The question amounts to this: would the conical orthomorphic projection provide a better plotting chart than the mercator? Over the small area covered by each chart, and particularly over the small local triangle formed by the D.R. position, the fix and the meridian of the D.R., the distortion should be negligible over the latitudes normally covered by surface navigation. The facility gained by the regular use of an unchanging scale of minutes of sextant altitude might be considerable. Faulty draughtsmanship in transferring minutes from a normal latitude scale at the side of a mercator chart might spoil much accurate work with almanac, tables and sextant.

I 2 Craigweil Avenue,

Manchester, 20.
Yours faithfully,

F. W. WRIGHT

\title{
THE VALUE OF MOON SIGHTS
}

SIR,-The contention in Mr. Burton's article (this Journal, Vol. V, p. 139) appears to be that because Moon sights are fairly frequently available at sea it follows that the Moon must be of great value as a position finding agent to those concerned with the navigation of merchant ships. This is not confirmed in practice for it is found that the weather conditions which favour an observation of the Moon will, almost certainly, ensure that the ship's position has been, or will shortly be, satisfactorily established by the normal observations of the Sun and stars which are matters of routine in a well-conducted merchant ship. The information supplied by the Moon is therefore merely redundant, unless of course, the normal routine is allowed to lapse.

In the higher latitudes in winter the longitude of the noon position as derived from the morning sight is often not very satisfactory on account of the small change of bearing, and it would seem that the noon position could with advantage be obtained by simultaneous sights of the Sun and Moon. Unfortunately, however, in high latitudes in winter the Moon is rarely high enough in the sky to be observed when on a suitable bearing at apparent noon. At other seasons of the year, and in low latitudes at all seasons, the Moon is astronomically available for the purpose for several days each half month; but then the longitude can be derived from the morning sight with all the accuracy required and the use of the Moon only delays the determination of the position.

Frequently enough at sea cloudy weather causes a failure, or partial failure, of our routine observations and then help from the Moon would be very welcome. Experience, however, tells us that help from that quarter is not at all likely to be forthcoming and that, when a break in the sky occurs, a sight of the Sun is the only thing worth hoping for.

To the seamen, then, the Moon sight is something which, very generally; can be obtained when it is not needed. Consequently he cannot use it very much and his valuation of the Moon as a position-finding agent is correspondingly low. The Moon is, however, found to be very useful for finding the error of the compass.

S.S. Ranchi,

At sea.
Yours faithfully,

C. F. HallidaY 